\title{
7.2. A PROPOSAL FOR A REFLECTING ASTROLABE
}

\author{
D. V. Thомаs* \\ (Royal Observatory, Cape, South Africa)
}

\begin{abstract}
A proposal is made for the construction of a $25 \mathrm{~cm}$ aperture "reflecting astrolabe", using a CERVIT prism of near-zero thermal expansion. Such an instrument could be expected to have an advantage over the Danjon astrolabe as regards accuracy, declination-range and limiting magnitude.

\section{RÉSUMÉ}

On propose de construire un "astrolabe à réflexion" de $25 \mathrm{~cm}$ d'ouverture, en utilisant un prisme de CER-VIT à coefficient de dilatation quasi nul. Un tel instrument serait supérieur a l'astrolabe Danjon en ce qui concerne la précision, la zône de déclinaisons accessible et la magnitude limite.
\end{abstract}

Although the quality of the results obtained with Danjon astrolabes is generally high, the instrument does suffer from some defects and limitations. The angle of the main prism has been found to be very sensitive to temperature gradients induced in the prism by changes in the ambient temperature. In general there is a systematic increase in prism angle as the instrument cools during a night's observing. The result is a large "closing error" in prism angle, making reliable determination of group corrections in prism angle impossible. The residual zenith distance of an individual star also depends critically on the rank of the star in the group. Stars observable with an individual astrolabe are limited to a band less than $60^{\circ}$ wide centred on the zenith. Thus the polar regions of the sky, particularly in the south, are not easily accessible. The limiting magnitude is about 6.0 in normal conditions, but the Paris (Guinot et al., 1961) and Herstmonceux (Thomas and Wallis, 1967) results show that the observations of stars within one magnitude of the limit become increasingly affected by colour and magnitude equations, which are not the same for all observers. These defects affect particularly the performance of the instrument for the determination of catalogue corrections, but they also introduce difficulties in the precise comparison of the time and latitude results obtained by observation of the same stars at different sites.

The recent development of the glass-ceramic material CER-VIT offers the possibility of overcoming most of the above difficulties. The coefficient of expansion of

* Now at Royal Greenwich Observatory, Hailsham, Sussex, England.

Markowitz and Guinot (eds.), Continental Drift, 105-107. () I.A.U. 
CER-VIT is more than a hundred times less than that of glass. Due to its crystalline structure, the material is generally unsuitable for transmission optics, but an aluminised CER-VIT prism, used as in Figure 1, could be expected to provide an altitude of observation stable to about. $\pm " 01$.

A CER-VIT prism is being obtained for an experimental modification of the Danjon astrolabe at the Royal Observatory, Cape. A preliminary design study has also been undertaken of a "reflecting astrolabe" (with CER-VIT prism) having a total aperture of $25 \mathrm{~cm}$, compared with $10 \mathrm{~cm}$ for the Danjon astrolabe (Thomas, 1967). The provisional layout of the optical components is illustrated in Figure 1, which may be compared with the corresponding diagram of the Danjon astrolabe (Danjon, 1958). The CER-VIT prism $(A)$ has an angle of $135^{\circ}$, for observing at an altitude of $45^{\circ}$. It could be replaced by one with a smaller angle, for observing at a greater altitude, without much difficulty. The $f / 10$ objective $(C)$ has a focal length of $2.5 \mathrm{~m}$. The quadruple Wollaston prism $(D)$ has the same angle as the one in the Danjon astrolabe, as the focal ratios are the same, but rather more than double the linear

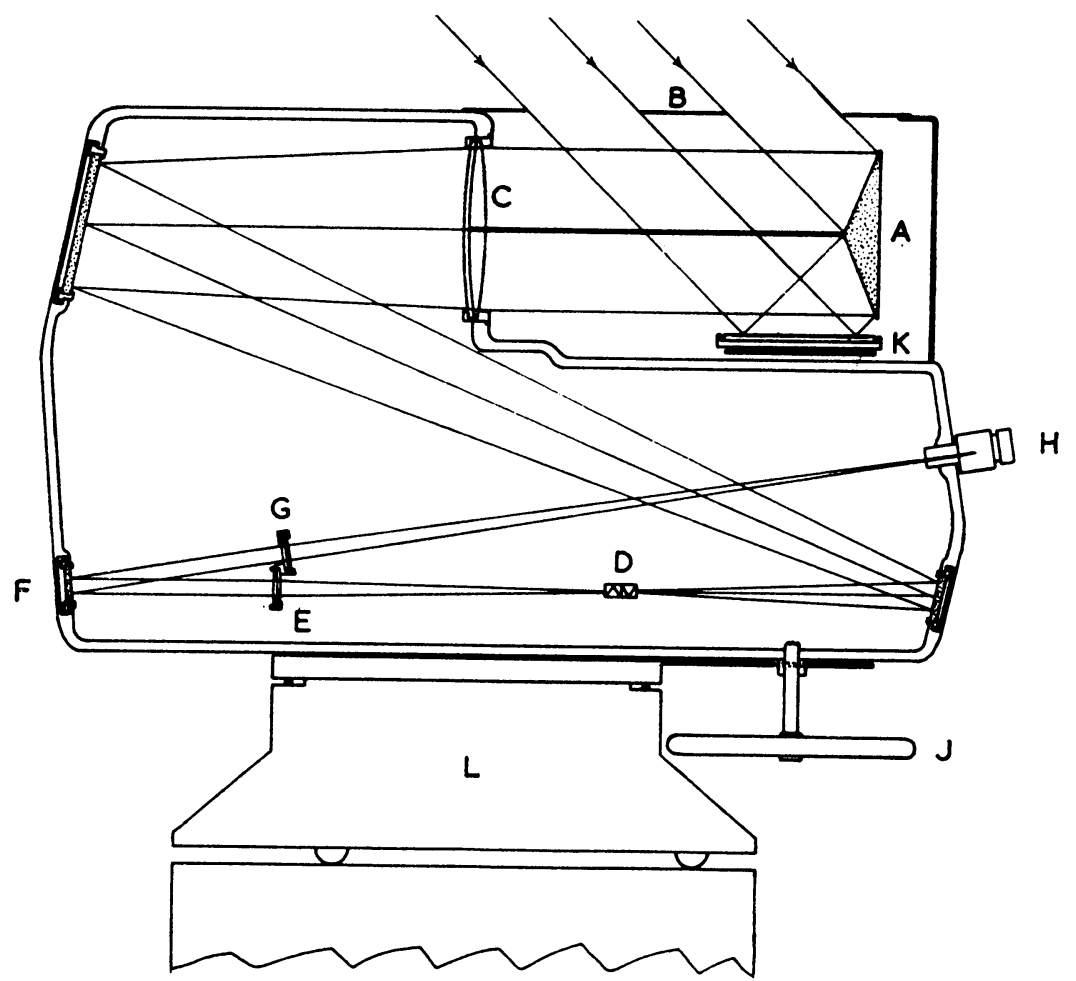

FIG. 1. Proposed $25 \mathrm{~cm}$ Reflecting Astrolabe with CER-VIT Prism. A, CER-VIT prism; B, removable wind-shield; $C, 25 \mathrm{~cm} \mathrm{f/10} \mathrm{objective;} D$, Wollaston prism; $E, G$, transfer lenses; $F$, plane mirror; $H$, orthoscopic eyepiece; $J$, "steering wheel"; $K$, mercury bath; L, base (schematic). 
dimensions. The run of the Wollaston prism allows the change of altitude of a star to be compensated for a minimum of \pm 20 s from the time of almucantar transit. The scale value of the micrometer is approximately $2^{\prime \prime}$ per millimetre run of the Wollaston prism. A nominal reading accuracy correct to 0.01 over the total range could easily be achieved by a commercial Moiré-fringe device using relatively coarse gratings, giving a digitised output direct on to punched cards or paper tape. The lens $(G)$ has a focal length twice that of $(E)$. The unwanted duplicated images formed by the Wollaston prism are removed by a stop placed immediately in front of $(G)$, at the position of the image of the objective formed by $(E)$. The $2.5 \mathrm{~cm}$ focal length orthoscopic eyepiece $(H)$ gives a final magnification of 200 , and a field of view $12^{\prime}$ in diameter. It is proposed that the "steering wheel" $(J)$ should enable a differential speed variation to be imparted direct to the carriage of the Wollaston prism. This would avoid the difficulty of a given angular displacement of the images in the field requiring an angular rotation of the steering wheel proportional to the cosecant of the azimuth of observation. The provision of an automatic slow-motion device in azimuth would enable the stellar images to be kept stationary in the middle of the field during the observation, and help to eliminate personal equations depending on the azimuth of the star observed.

The detailed mechanical layout of the instrument has not yet been considered, but its construction should not present any insuperable difficulties.

In good conditions the proposed reflecting astrolabe could be expected to be more accurate than the Danjon astrolabe, due to greater stability of the altitude of observation, the slightly higher magnification (200 as against 175), and the smaller Airy disk (principal axes of first dark refraction ring $2.5 \times 1.5$ ). It could operate at any selected altitude above $45^{\circ}$, enabling both north and south poles to be reached from existing stations. The limiting magnitude of about 8.0 would bring all the stars of the FK4 and the majority of the stars of the GC within range. The brighter minor planets would be observable for varying periods from any station, providing a valuable link between the astrolabe system of stellar coordinates and the dynamical theory of the solar system.

\section{References}

Danjon, A. (1958) M.N.R.A.S., 118, 411.

Guinot, B., Débarbat, S., Krieger-Fiel, J. (1961) Bull. astr., 23, 307.

Thomas, D.V. (1967) Mon. Notes astr. Soc. Sth. Afr., 26, 2.

Thomas, D.V., Wallis, R.E. (1967) R. Obs. Bull. in preparation. 